\title{
The relationship between lymphovascular invasion and angiogenesis, hormone receptors, cell proliferation and survival in patients with primary operable invasive ductal breast cancer
}

Zahra MA Mohammed ${ }^{1,2^{*}}$, Donald C McMillan ${ }^{1 \dagger}$, Joanne Edwards ${ }^{3 \dagger}$, Elizabeth Mallon ${ }^{4 \dagger}$, Julie C Doughty ${ }^{5 \dagger}$, Clare Orange $^{4 \dagger}$ and James J Going ${ }^{3 \dagger}$

\begin{abstract}
Background: Several well-established tumour prognostic factors are used to guide the clinical management of patients with breast cancer. Lymphovascular invasion and angiogenesis have also been reported to have some promise as prognostic factors. The aim of the present study was to examine the prognostic value of tumour lymphovascular invasion and microvessel density compared with that of established prognostic factors in invasive ductal breast cancer.

Methods: In addition to hormone receptor status and Ki-67 proliferative activity, lymphovascular invasion and microvessel density and their relationship with survival were examined in patients with invasive ductal breast cancer. Full sections and tissue microarrays ( $n=384$ patients) were utilised to assess these factors and were scored by appropriate methods.

Results: On univariate analysis tumour size $(P<0.05)$, lymph node involvement $(P<0.01)$, lymphovascular invasion $(P<0.05)$, microvessel density $(P<0.05)$ and local- regional treatment $(P<0.01)$ were associated with poorer survival in ER negative tumours. On multivariate analysis in ER negative tumours lymph node involvement $(P<0.01)$ and local- regional treatment $(P<0.05)$ were independently associated with poorer cancer-specific survival. On univariate analysis tumour grade $(P<0.05)$, lymph node involvement $(P<0.001)$, HER-2 $(P<0.05)$, Ki-67 $(P<0.01)$ and lymphovascular invasion $(P<0.001)$ were associated with poorer survival in ER positive tumours. On multivariate analysis lymph node involvement $(P<0.001)$, Ki-67 $(P<0.001)$ and lymphovascular invasion $(P<0.05)$ were independently associated with poorer cancer-specific survival in ER positive tumours.
\end{abstract}

Conclusion: Lymphovascular invasion but not microvessel density was independently associated with poorer survival in patients with ER positive but not ER negative invasive ductal breast cancer.

Keywords: Primary invasive breast cancer, Prognostic factors, Lymphovascular invasion, Angiogenesis, Survival

\footnotetext{
* Correspondence: mrxzma@hotmail.com

${ }^{\dagger}$ Equal contributors

${ }^{1}$ Academic Unit of Surgery, College of Medical, Veterinary and Life of

Sciences, Royal Infirmary, University of Glasgow, Glasgow G31 2ER, UK

${ }^{2}$ University Departments of Pathology, Faculty of Veterinary Medicine, Omar

Almukhtar University, Al bayda, PO Box 919, Libya

Full list of author information is available at the end of the article
} 


\section{Background}

Breast cancer is the commonest cancer and the leading cause of cancer death in women accounting for $22 \%$ of all female cancers [1]. Although approximately $80 \%$ of the 42,000 women in the UK are diagnosed with breast cancer each year and survive at least five years [2], it is still the leading cause of cancer death in women.

Prognostic factors aid clinical decision making, treatment selection for individual patients and allow comparisons between groups of patients at risk of recurrence or death. Clinically useful prognostic/predictive factors should have biological relevance, be reproducible in different laboratories, be validated prospectively in large series of patients, be confirmed independently by other workers and have threshold levels that are already optimized [3].

With reference to breast cancer, well-established clinicopathological indicators of clinical outcome and response to therapy are age, histologic type, grade, tumour size, lymph node status and hormone receptor expression [4,5]. Expression of oestrogen receptor (ER) and progesterone receptor (PR) is associated with better survival and response to oestrogen competive agonists such as tamoxifen, independently of other variables [4,6-10]. More recently, human epidermal growth factor receptor 2 (HER-2) status has become an established clinicopathological indicator of breast cancer clinical outcome and response to therapy $[4,5]$. Proliferation is recognised to be a key feature of tumour progression and is now widely estimated by the nuclear antigen Ki-67, which is tightly linked to the cell cycle. Several recent studies have reported an association between higher Ki-67 proliferative activity and poorer recurrence-free [11-13] and cancer-specific survival [14-16].

Lymphovascular invasion is a crucial step in the complex process of tumour metastasis and an important criterion for further therapy. The presence of carcinoma cells in either lymphatic vessels (lymphatic invasion), blood vessels (vascular invasion) or both (lymphovascular invasion) is a significant prognostic factor in invasive breast cancer, with respect to local and distance recurrence [17-22] and poorer survival [19-25]. At the St. Gallen meeting in 2005, lymphovascular invasion was recognised as a prognostic factor for node-negative patients [26]. Node-negative patients with lymphovascular invasion had higher breast cancer mortality rate (53\%) compared with patients with no lymphovascular invasion (29\%) [27]. Lymphovascular invasion is also associated with other strongest prognostic factors including tumour size, grade and loco- regional lymph node involvement $[21,27,28]$.

The role of angiogenesis (development of new capillaries from pre-existing vessels) in the growth of solid tumours is recognized [29]. Furthermore, it is a vital not only for the development and progression of primary carcinomas but also for invasion and metastasis of solid tumours [30]. Accumulating evidence indicate that progressive tumour growth is dependent on angiogenesis [31] and several studies have reported an association between microvessel density and poorer recurrence- free [23,32-37], cancer- specific survival [16] and overall survival [16,23,32,34,35,38]. Microvessel density is also associated with a poorer clinical response to chemotherapy [39] and to endocrine therapy $[39,40]$. On the other hand, some authors do not find any association between microvessel density and recurrence- free survival and overall survival $[41,42]$ and one found a direct association between microvessel density and clinical response to chemotherapy [43]. To date there have been few studies that have directly compared these different approaches and their relationship with outcome. Moreover, it is not clear whether angiogenesis, as measured by microvessel density, may add additional prognostic information to established prognostic and predictive factors. Therefore, the aim of the present study was to examine the prognostic value of lymphovascular invasion and microvessel density and compare it with other well-established clinicopathological prognostic factors.

\section{Methods}

Patients presenting with invasive breast cancer at Glasgow Royal and Western Infirmaries and Stobhill Hospital, all in the West of Scotland, between 1995 and 1998 and who had lymphovascular invasion assessed were studied $(\mathrm{n}=384)$. Available clinicopathological data included age, histological tumour type, grade, tumour size, lymph node status, type of surgery and use of adjuvant treatment (chemotherapy, hormonal therapy and/or radiotherapy).

The study was approved by the Research Ethics Committee of the North Glasgow University Hospitals NHS Trust.

\section{Tissue micro array (TMA) construction}

Tissue microarrays (TMA) were used in this study. In brief, each specimen had tumour rich area identified by a pathologist and 3 cores $0.6-\mathrm{mm}^{2}$ tumour cores were used to construct the TMA [44].

\section{Assessment for tumour ER, PR and HER-2 statuses and Ki-67 proliferative index}

Tissue microarrays (TMA) were utilized to assess Oestrogen (ER), progesterone (PR) status and HER-2 status as previously described $[45,46]$ and to assess Ki-67 proliferative activity as previously described [47]. Also TMA were used to assess microvessel density by immunohistochemistry for CD34 +.

\section{Immunohistochemistry for microvessel density}

In addition to TMA, Immunoreactivity to anti-CD34+ antibody was determined in 33 cases by using full-face 
tissue sections. Freshly cut $2.5 \mu \mathrm{m}$ thick breast cancer full-face tissue sections and TMAs were stained for CD34+. Sections and cores were dewaxed and rehydrated for antigen heat-retrieval in Tris EDTA buffer ( $\mathrm{pH} 8$ ), under pressure for 7 minutes. Endogenous peroxidise was blocked by incubation in 3\% hydrogen peroxide for 20 minutes. Arrays were then incubated in normal horse serum 1:10 for 30 minutes at $25^{\circ} \mathrm{C}$ to block non- specific binding. The primary antibody (Monoclonal mouse antihuman, CD34+ Class II, Clone QBEnd 10, Code M7165, DAKO, Glostrup, Denmark) dilutied 1:150 was applied for 30 minutes at $25^{\circ} \mathrm{C}$ with detection using Envision (DAKO code K5007, Glostrup, Denmark) and DAB (3-3' diaminobenzidine, Vector code SK 4001, USA) according to manufacturer's instruction. Arrays were lightly counterstained with haematoxylin, dehydrated and mounted in DPX. Sections of tonsil were used as positive and negative controls for each staining batch as tonsil has many CD34+ cells.

\section{Slide scanning and scoring}

Stained slides were scanned using a Hamamatsu NanoZoomer (Hertfordshire, UK). Visual counting of vessel was performed on a computer monitor.

\section{Lymphovascular invasion}

Lymphovascular invasion was assessed by using a hematoxylin and eosin on full sections. Blood and lymphatic vessel invasion was not distinguished. Lymphovascular invasion was recorded as present or absent.

\section{Assessment of tumour microvessel density}

Microvessel counting was a modification of the method described by Weidner et al. (1992) in which large microvessels as well as any single stained endothelial cells clearly separated from adjacent microvessels, tumour cells, and other connective tissue elements were considered single, countable microvessels. Branching structures were counted as one, unless there was a break in continuity, in which case it was counted as two distinct vessels [37].

The mean vessel count for the three cores of each tumour sample was used for subsequent analysis. One hundred eighty nine cores, counted independently by two observers (C.O. and Z.M.) blinded to patient outcome and the other observer's score, yielded an interclass correlation coefficient (ICCC) of 0.96, indicating excellent agreement. Z.M. subsequently scored all slides. Accuracy of scoring depends on individual cores containing a satisfactory sample of tumour cells, which was checked by a qualified pathologist (J.J.G.). As there are no universally accepted prognostic thresholds for microvessel counts $(\mathrm{CD} 34+)$, survival analysis was undertaken using tertiles. In the full-face tissue sections, CD34+ was counted in three $\mathrm{x} 40$ fields and the mean for the three fields was calculated. The mean for the three fields in full-face sections was compared with the mean of three cores in TMA for the same patients. The ICCC was 0.87, which indicates excellent agreement.

\section{Statistical analysis}

Inter-relationships between variables were assessed using contingency tables with the chi-squared test for trend as appropriate. Univariate analysis and multivariate survival analysis with calculation of hazard ratios (HR) were performed using Cox's proportional-hazards model. A stepwise backward procedure was used to derive a final model of the variables that had a significant independent relationship with survival. Deaths up to March 2010 were included in the analysis. Inter-relationships between methods were assessed using contingency table analysis with the chi-squared test for trend as appropriate. Because of the number of statistical comparisons, a $P$ value $<0.01$ was considered to be significant All statistical analysis was performed using SPSS software version 19 (SPSS Inc., Chicago, IL, USA).

\section{Results}

The clinicopathological characteristics of 384 patients with primary operable breast cancer are shown in Table 1.

The relationship between ER status and clinicopathological characteristics is shown in Table 2. Patients with ER negative tumours were younger $(P<0.01)$, had larger carcinomas $(P<0.01)$, of higher tumour grade $(P<0.001)$, more likely to be PR negative tumours $(P<0.001)$, more likely to be HER-2 positive tumours $(P<0.001)$, with more lymphovascular invasion $(P<0.001)$. They were also more likely to receive systemic adjuvant treatment in the form of chemotherapy $(P<0.001)$ and had a shorter cancer- specific survival $(P<0.001)$.

The minimum follow-up was 142 months; the median follow-up of the survivors was 165 months. In the patients with ER negative tumours 58 patients developed recurrence, 8 local, 38 distant and 4 both, 79 patients died, 48 of their disease. In the ER positive tumours, 46 patients developed recurrence, 9 local, 31 distant and 1 both, 120 patients died, 47 of their disease.

The relationship between clinico-pathological characteristics of patients with ER negative primary operable invasive ductal breast cancer and recurrence- free survival was examined. On univariate survival analysis tumour size (HR 2.70, 95\% CI 1.43-5.09, $P=0.002$ ), lymph node involvement (HR 1.71, 95\% CI 1.17-2.50, $P=0.006$ ), lymphovascular invasion (HR 2.71, 95\% CI 1.34-5.50, $P=0.006$ ), microvessel density (HR 1.57, 95\% CI 1.06-2.34, $P=0.026)$ and local-regional treatment (HR 2.29, 95\% CI 1.08-4.87, $P=0.032$ ) were significantly associated with recurrence- free survival. On multivariate survival analysis, tumour size (HR 2.27, 95\% CI 1.19-4.36, 
Table 1 The clinico-pathological characteristics of patients with primary operable invasive ductal breast cancer $(\mathrm{n}=$ 384)

\begin{tabular}{ll}
\hline Clinico-pathological characteristics & Patients (n) \\
\hline Age $(\leq 50 />50$ years) & $107(28 \%) / 277(72 \%)$ \\
Size $(\leq 20 / 21-50 / \geq 50 \mathrm{~mm})$ & $218(57 \%) / 154(40 \%) / 11(3 \%)$ \\
Grade (I/I/III) & $70(18 \%) / 157(41 \%) / 157(41 \%)$ \\
Involved lymph node (0/1-3/>3) & $205(53 \%) / 108(28 \%) / 66(17 \%)$ \\
Oestrogen -receptor status (ER-/ER+) & $124(32 \%) / 237(62 \%)$ \\
Progesterone -receptor status (PR-/PR+) & $193(50 \%) / 170(44 \%)$ \\
HER-2 status (HER-2 -/HER-2+) & $299(78 \%) / 65(17 \%)$ \\
Ki-67 status (Low Ki-67/High Ki-67) & $272(71 \%) / 92(24 \%)$ \\
LVI (Absent/Present) & $234(61 \%) / 150(39 \%)$ \\
MVD (CD34+) (tertiles 1, 2, 3) & $137(36 \%) / 126(33 \%) / 106(28 \%)$ \\
Loco-regional treatment (Lumpectomy + radiotherapy/mastectomy + radiotherapy) & $142(37 \%) / 242(63 \%)$ \\
Systemic treatment (ER-based treatment) (hormonal/hormonal + chemotherapy/chemotherapy/none) & $192(50 \%) / 86(22 \%) / 80(21 \%) / 22(6 \%)$ \\
\hline
\end{tabular}

$P=0.013)$ and lymphovascular invasion (HR 2.35, 95\% CI 1.10-5.01, $P=0.028$ ) were independently associated with recurrence- free survival.

The relationship between clinicopathological characteristics of patients with ER negative primary operable invasive ductal breast cancer and cancer- specific survival is shown in Table 3 . On univariate survival analysis tumour size $(P<0.05)$, lymph node involvement $(P<0.01)$, lymphovascular invasion $(P<0.05)$, microvessel density $(P<0.05)$ and loco-regional treatment $(P<0.01)$ were significantly associated with cancerspecific survival. On multivariate survival analysis, lymph node involvement $(P<0.01)$ and loco-regional treatment $(P<0.05)$ were independently associated with cancerspecific survival.
The relationship between clinicopathological characteristics of patients with ER positive primary operable invasive ductal breast cancer and recurrence- free survival was examined. On univariate survival analysis, tumour grade (HR 1.89, 95\% CI 1.12-3.17, $P=0.017$ ), lymph node involvement (HR 2.42, 95\% CI 1.55-3.76, $P<0.001$ ), HER2 status (HR 2.90, 95\% CI 1.25-6.72, $P=0.013$ ), Ki-67 proliferative activity (HR 3.01, 95\% CI 1.50-6.04, $P=0.002$ ), lymphovascular invasion (HR 3.83, 95\% CI 1.89-7.77, $P \leq 0.001$ ), microvessel density (HR 1.70, 95\% CI 1.05-2.73, $P=0.030)$ and systemic treatment (HR 1.59, 95\% CI $1.09-2.32, P=0.017)$ were significantly associated with recurrence- free survival. On multivariate survival analysis, lymph node involvement (HR 2.15, 95\% CI 1.35-3.44, $P=0.001$ ) and Ki-67 proliferative activity (HR

Table 2 The relationship between clinico-pathological characteristics and ER status of patients with primary operable invasive ductal breast cancer

\begin{tabular}{|c|c|c|c|}
\hline & $\begin{array}{l}\text { Oestrogen receptor } \\
\text { negative }(n=124)\end{array}$ & $\begin{array}{l}\text { Oestrogen receptor } \\
\text { positive }(n=237)\end{array}$ & (P-value) \\
\hline Age $(\leq 50 />50$ years $)$ & $43 / 81$ & $52 / 185$ & 0.009 \\
\hline Size $(\leq 20 / 21-50 />50 \mathrm{~mm})$ & $57 / 62 / 4$ & $149 / 83 / 5$ & 0.004 \\
\hline Grade $(I / I / I I I)$ & $3 / 22 / 99$ & $61 / 125 / 51$ & $<0.001$ \\
\hline Involved lymph node (0/1-3/>3) & $65 / 30 / 28$ & $130 / 68 / 35$ & 0.211 \\
\hline Progesterone -receptor status (PR-/PR+) & $117 / 5$ & $72 / 161$ & $<0.001$ \\
\hline HER-2 status (HER-2 -/HER-2+) & $80 / 41$ & $212 / 24$ & $<0.001$ \\
\hline Ki-67 status (Low Ki-67/High Ki-67) & $99 / 24$ & $160 / 68$ & 0.036 \\
\hline LVI (Absent/Present) & $56 / 68$ & $161 / 76$ & $<0.001$ \\
\hline MVD (CD34+) (tertiles 1, 2, 3) & $38 / 41 / 44$ & $83 / 84 / 61$ & 0.106 \\
\hline Loco-regional treatment (Lumpectomy + radiotherapy/mastectomy + radiotherapy) & $44 / 80$ & $89 / 148$ & 0.699 \\
\hline $\begin{array}{l}\text { Systemic treatment (ER-based treatment) (hormonal/hormonal + } \\
\text { chemotherapy/chemotherapy/none) }\end{array}$ & 26/19/66/11 & $158 / 61 / 8 / 8$ & $<0.001$ \\
\hline Cancer specific survival (months) ${ }^{*}$ & $130(118-142)$ & $156(150-162)$ & 0.001 \\
\hline
\end{tabular}


Table 3 The relationship between clinico-pathological characteristics of patients with ER negative primary operable invasive ductal breast cancer and cancer- specific survival

\begin{tabular}{|c|c|c|c|c|}
\hline & \multirow{2}{*}{\multicolumn{2}{|c|}{$\begin{array}{c}\text { Univariate survival analysis } \\
\text { Cancer-specific survival }\end{array}$}} & \multirow{2}{*}{\multicolumn{2}{|c|}{$\begin{array}{c}\text { Multivariate survival analysis } \\
\text { Cancer-specific survival }\end{array}$}} \\
\hline & & & & \\
\hline & $\begin{array}{c}\text { Hazard ratio } \\
(95 \% \mathrm{Cl})\end{array}$ & $P$-value & $\begin{array}{c}\text { Hazard ratio } \\
(95 \% \mathrm{Cl})\end{array}$ & $P$-value \\
\hline Age $(<50 />50$ years $)$ & $0.78(0.42-1.47$ & 0.448 & & \\
\hline Size $(\leq 20 / 21-50 />50 \mathrm{~mm})$ & $2.19(1.14-4.22)$ & 0.018 & & 0.116 \\
\hline Grade $(I / I / I I I)$ & $1.30(0.63-2.72)$ & 0.478 & & \\
\hline Involved lymph node (0/1-3/>3) & $1.85(1.24-2.66)$ & 0.001 & $1.71(1.17-2.50)$ & 0.006 \\
\hline Progesterone -receptor status (PR-/PR+) & $0.05(0.00-22.73)$ & 0.330 & & \\
\hline HER-2 status (HER-2 -/HER-2+) & $1.26(0.66-2.41)$ & 0.489 & & \\
\hline Ki-67 status (Low Ki-67/High Ki-67) & $1.48(0.70-3.11)$ & 0.305 & & \\
\hline LVI (Absent/Present) & $2.14(1.08-4.22)$ & 0.029 & & 0.428 \\
\hline MVD (CD34+) (tertiles 1, 2, 3) & $1.62(1.08-2.44)$ & 0.021 & & 0.130 \\
\hline $\begin{array}{l}\text { Loco-regional treatment (Lumpectomy + radiotherapy/ } \\
\text { mastectomy + radiotherapy) }\end{array}$ & $3.27(1.44-7.42)$ & 0.005 & $2.64(1.14-6.09)$ & 0.023 \\
\hline $\begin{array}{l}\text { Systemic treatment (ER-based treatment) (hormonal/ } \\
\text { hormonal + chemotherapy/chemotherapy/none) }\end{array}$ & $1.00(0.70-1.42)$ & 1.00 & & \\
\hline
\end{tabular}

3.49, 95\% CI 1.51-8.07, $P=0.003)$ were independently associated with recurrence- free survival.

The relationship between clinicopathological characteristics of patients with ER positive primary operable invasive ductal breast cancer and cancer- specific survival is shown in Table 4. On univariate survival analysis tumour size $(P<0.01)$, tumour grade $(P<0.05)$, lymph node involvement $(P<0.001)$, Ki-67 proliferative activity $(P<0.001)$, lymphovascular invasion $(P<0.001)$ and systemic treatment $(P<0.05)$ were significantly associated with cancer- specific survival. On multivariate survival analysis, lymph node involvement $(P<0.01)$, Ki-67 proliferative activity $(P<$ $0.001)$ and lymphovascular invasion $(P<0.05)$ were independently associated with cancer- specific survival.

The inter-relationships between clinicopathological characteristics for patients with ER negative primary operable invasive ductal breast cancer are shown in Table 5. Age was negatively associated PR status $(P<0.01)$. Increased tumour size was positively associated with more involved lymph node $(P<0.01)$. Involved lymph node was positively

Table 4 The relationship between clinico-pathological characteristics of patients with ER positive primary operable invasive ductal breast cancer and cancer- specific survival

\begin{tabular}{|c|c|c|c|c|}
\hline & \multirow{2}{*}{\multicolumn{2}{|c|}{$\begin{array}{c}\text { Univariate survival analysis } \\
\text { Cancer-specific survival }\end{array}$}} & \multirow{2}{*}{\multicolumn{2}{|c|}{$\begin{array}{c}\text { Multivariate survival analysis } \\
\text { Cancer-specific survival }\end{array}$}} \\
\hline & & & & \\
\hline & $\begin{array}{l}\text { Hazard ratio } \\
(95 \% \mathrm{Cl})\end{array}$ & $P$-value & $\begin{array}{l}\text { Hazard ratio } \\
(95 \% \mathrm{Cl})\end{array}$ & $P$-value \\
\hline Age (<50/>50 years) & $1.82(0.77-4.33)$ & 0.173 & & \\
\hline Size $(\leq 20 / 21-50 />50 \mathrm{~mm})$ & $2.04(1.20-3.49)$ & 0.009 & & 0.116 \\
\hline Grade $(I / I / \mid I I I)$ & $1.71(1.09-2.68)$ & 0.019 & & 0.342 \\
\hline Involved lymph node (0/1-3/>3) & $2.26(1.54-3.32)$ & $<0.001$ & $2.11(1.40-3.20)$ & $<0.001$ \\
\hline Progesterone -receptor status (PR-/PR+) & $0.72(0.38-1.37)$ & 0.316 & & \\
\hline HER-2 status (HER-2 -/HER-2+) & $2.17(0.96-4.88)$ & 0.062 & & 0.109 \\
\hline Ki-67 status (Low Ki-67/High Ki-67) & $3.71(1.98-6.96)$ & $<0.001$ & $3.62(1.88-7.00)$ & $<0.001$ \\
\hline LVI (Absent/Present) & $2.98(1.62-5.47)$ & $<0.001$ & $2.36(1.22-4.58)$ & 0.011 \\
\hline MVD (CD34+) (tertiles 1, 2, 3) & $1.41(0.95-2.08)$ & 0.087 & & 0.667 \\
\hline $\begin{array}{l}\text { Loco-regional treatment (Lumpectomy + radiotherapy/ } \\
\text { mastectomy + radiotherapy) }\end{array}$ & $1.75(0.90-3.43)$ & 0.100 & & \\
\hline $\begin{array}{l}\text { Systemic treatment (ER-based treatment) (hormonal/ } \\
\text { hormonal + chemotherapy/chemotherapy/none) }\end{array}$ & $1.45(1.03-2.05)$ & 0.033 & & 0.481 \\
\hline
\end{tabular}


Table 5 Inter-relationships between the clinicopathological characteristics in patients with ER negative primary operable invasive ductal breast cancer $(n=124)$

\begin{tabular}{|c|c|c|c|c|c|c|c|c|c|c|}
\hline & $\begin{array}{l}\text { Size } \\
\text { ( } P \text {-value) }\end{array}$ & $\begin{array}{l}\text { Grade } \\
\text { (P-value) }\end{array}$ & $\begin{array}{l}\text { Involved } \\
\text { lymph node } \\
(P \text {-value })\end{array}$ & $\begin{array}{l}\text { PR } \\
\text { status } \\
\text { (P-value) }\end{array}$ & $\begin{array}{l}\text { HER-2 } \\
\text { status } \\
\text { (P-value) }\end{array}$ & $\begin{array}{l}\text { Ki-67 } \\
\text { status } \\
\text { (P-value) }\end{array}$ & $\begin{array}{l}\text { LVI } \\
\text { (P-value) }\end{array}$ & $\begin{array}{l}\text { MVD } \\
\text { (P-value) }\end{array}$ & $\begin{array}{l}\text { Loco-regional } \\
\text { treatment } \\
(P \text {-value })\end{array}$ & $\begin{array}{l}\text { Systemic } \\
\text { treatment } \\
(P \text {-value })\end{array}$ \\
\hline Age ( $\leq 50 />50$ years) & 0.241 & 0.908 & 0.242 & 0.002 & 0.154 & 0.772 & 0.826 & 0.347 & 0.282 & 0.059 \\
\hline Size $(\leq 20 / 21-50 />50 \mathrm{~mm})$ & & 0.181 & 0.004 & 0.131 & 0.343 & 0.085 & 0.011 & 0.106 & 0.042 & 0.151 \\
\hline Grade $(I / I / I I I)$ & & & 0.127 & 0.049 & 0.730 & 0.586 & 0.042 & 0.586 & 0.980 & 0.164 \\
\hline $\begin{array}{l}\text { Involved lymph node } \\
(0 / 1-3 />3)\end{array}$ & & & & 0.166 & 0.058 & 0.392 & $<0.001$ & 0.068 & 0.013 & 0.118 \\
\hline $\begin{array}{l}\text { Progesterone -receptor } \\
\text { status (PR-/PR+) }\end{array}$ & & & & & 0.769 & 0.985 & 0.111 & 0.191 & 0.093 & 0.272 \\
\hline $\begin{array}{l}\text { HER-2 status } \\
\text { (HER-2 -/HER-2+) }\end{array}$ & & & & & & 0.949 & 0.001 & 0.340 & 0.068 & 0.292 \\
\hline $\begin{array}{l}\text { Ki-67 proliferative activity } \\
\text { (Low Ki-67/High Ki-67) }\end{array}$ & & & & & & & 0.739 & 0.651 & 0.256 & 0.335 \\
\hline LVI (Absent/Present) & & & & & & & & 0.414 & 0.054 & 0.563 \\
\hline MVD (tertiles 1, 2, 3) & & & & & & & & & 0.020 & 0.944 \\
\hline $\begin{array}{l}\text { Loco-regional treatment } \\
\text { (Lumpectomy + radiotherapy/ } \\
\text { mastectomy + radiotherapy) }\end{array}$ & & & & & & & & & & 0.432 \\
\hline
\end{tabular}

Bold data reflects an association between the two variables.

associated with the presence of lymphovascular invasion $(P<0.001)$. HER-2 status was positively associated with the presence of lymphovascular invasion $(P<0.01)$.

The inter-relationships between clinicopathological characteristics for patients with ER positive primary operable invasive ductal breast cancer are shown in Table 6 .
Age was negatively associated receiving systemic treatment $(P<0.001)$. Increased tumour size was positively associated with more involved lymph node $(P<0.001)$, the presence of lymphovascular invasion $(P<0.001)$, and locoregional treatment $(P<0.001)$. Higher tumour grade was positively associated with HER-2 + status $(P<0.001)$, Ki-67

Table 6 Inter-relationships between the clinicopathological characteristics in patients with ER positive primary operable invasive ductal breast cancer $(n=237)$

\begin{tabular}{|c|c|c|c|c|c|c|c|c|c|c|}
\hline & $\begin{array}{l}\text { Size } \\
\text { (P-value) }\end{array}$ & $\begin{array}{l}\text { Grade } \\
\text { (P-value) }\end{array}$ & $\begin{array}{l}\text { Involved } \\
\text { lymph } \\
\text { node } \\
(P \text {-value })\end{array}$ & $\begin{array}{l}\text { PR } \\
\text { status } \\
\text { (P-value) }\end{array}$ & $\begin{array}{l}\text { HER-2 } \\
\text { status } \\
\text { (P-value) }\end{array}$ & $\begin{array}{l}\text { Ki-67 } \\
\text { status } \\
\text { (P-value) }\end{array}$ & (P-value) & $\begin{array}{l}\text { MVD } \\
\text { (P-value) }\end{array}$ & $\begin{array}{l}\text { Loco-regional } \\
\text { treatment } \\
\text { (P-value) }\end{array}$ & $\begin{array}{l}\text { Systemic } \\
\text { treatment } \\
(P \text {-value })\end{array}$ \\
\hline Age ( $\leq 50 />50$ years $)$ & 0.058 & 0.236 & 0.495 & 0.297 & 0.054 & 0.641 & 0.913 & 0.238 & 0.261 & $<0.001$ \\
\hline Size $(\leq 20 / 21-50 />50 \mathrm{~mm})$ & & 0.033 & $<0.001$ & 0.879 & 0.826 & 0.041 & $<0.001$ & 0.568 & $<0.001$ & 0.438 \\
\hline Grade $(|/||/| I \mid)$ & & & 0.258 & 0.753 & $<0.001$ & $<0.001$ & $<0.001$ & 0.072 & 0.108 & 0.627 \\
\hline $\begin{array}{l}\text { Involved lymph } \\
\text { node }(0 / 1-3 />3)\end{array}$ & & & & 0.157 & 0.323 & 0.035 & $<0.001$ & 0.607 & 0.001 & 0.013 \\
\hline $\begin{array}{l}\text { Progesterone -receptor } \\
\text { status (PR-/PR+) }\end{array}$ & & & & & 0.002 & 0.340 & 0.392 & 0.997 & 0.256 & 0.804 \\
\hline $\begin{array}{l}\text { HER-2 status } \\
\text { (HER-2 -/HER-2+) }\end{array}$ & & & & & & 0.020 & 0.119 & 0.627 & 0.363 & 0.603 \\
\hline $\begin{array}{l}\text { Ki-67 proliferative activity } \\
\text { (Low Ki-67/High Ki-67) }\end{array}$ & & & & & & & 0.049 & 0.041 & 0.598 & 0.289 \\
\hline LVI (Absent/Present) & & & & & & & & 0.215 & 0.031 & 0.255 \\
\hline MVD (tertiles 1, 2, 3) & & & & & & & & & 0.628 & 0.402 \\
\hline $\begin{array}{l}\text { Loco-regional treatment } \\
\text { (Lumpectomy + radiotherapy/ } \\
\text { mastectomy + radiotherapy) }\end{array}$ & & & & & & & & & & 0.057 \\
\hline
\end{tabular}


proliferative activity $(P<0.001)$ and the presence of lymphovascular invasion $(P<0.001)$. Involved lymph node was positively associated with the presence of lymphovascular invasion $(P<0.001)$ and loco-regional treatment $(P<0.01)$. The presence of $P R$ was negatively associated with HER-2 + status $(P<0.01)$. Ki-67 proliferative activity was positively associated with microvessel density $(P<0.01)$.

\section{Discussion}

The results of the present study showed that lymphovascular invasion but not microvessel density was consistently associated with poorer recurrence- free and cancer-specific survival in both ER negative and ER positive tumours. The results of the present study also confirmed that established tumour characteristics such as tumour size, grade, nodal status, hormone status and Ki-67 proliferative activity provide prognostic value. Therefore, in the context of the present comprehensive examination of the prognostic value of tumour pathological features, it can be concluded that lymphovascular invasion may have an important role in determining outcome in patients with primary operable invasive ductal breast cancer.

The results of the present study are consistent with the previous studies that reported prognostic value of the lymphovascular invasion independent of involvement lymph node as well as other tumour characteristics such as grade, PR and HER-2 status [19-22,25]. However, some studies reported that the presence of LVI was not independently associated with outcome in primary breast cancer $[28,48]$ and others reported no association [49,50].

The results of the present study are also consistent with the previous studies that reported an association between the microvessel density and poorer survival $[16,23,32-38]$. However, in the present study it was of interest that microvessel density was associated with poorer survival in ER negative but not ER positive tumours whereas lymphovascular invasion was associated with poorer survival in both ER negative and ER positive tumours. Therefore, it would appear that lymphovascular invasion process is more consistently associated with poor outcome in patients with primary breast cancer.

The results of the present study are consistent with the Hayes and co-workers that concluded microvessel density alone could not be recommended as a basis for clinical decision making [51]. However, Kato and coworkers (2003) concluded that microvessel density, by various methods, did offer additional prognostic value to lymphovascular invasion and provided more reliable prognostic information than lymph node status [24].

In this context, it was of interest that in the present study there was an association between increased lymphovascular invasion and lymph node involvement in both ER negative and ER positive tumours. Also, in patients with ER positive tumours there was an association between lymphovascular invasion, tumour size and tumour grade. In contrast, angiogenesis (MVD) was only associated with Ki-67 proliferative activity. Taken together, these results are consistent with the hypothesis a proliferating tumour promotes angiogenesis, lymphovascular invasion, nodal involvement and metastases and then subsequent poor survival. Therefore, it may be that angiogenesis is essential for tumour growth whereas lymphovascular invasion is essential for the tumour to metastasise. Consistent with this hypothesis, in the smallest tumours $(<10 \mathrm{~mm}, \mathrm{n}=66)$ angiogenesis was observed in $83 \%$ of patients and lymphovascular invasion was observed in $29 \%$ of patients. In contrast, nodal involvement was only present in $23 \%$ of patients. The basis of these associations of lymphovascular invasion, angiogenesis and poorer cancer outcome in ductal breast cancer is not clear but may involve the tumour inflammatory cell infiltrate [52]. Therefore, further work is required to examine these potential relationships. Irrespective, it is apparent that lymphovascular invasion and angiogenesis are not isolated pathological features but are strongly related to other aggressive tumour characteristics. To minimize the risk of measurement bias, two independent observers examined the same cores before assigning an overall score. High levels of independent inter-observer agreement in the assessment of CD34+ suggest that this technique is reliable.

There is limitation of whether a small core sample used in the present TMA was representative of the entire tumour was examined, using a pilot comparison study of at 33 full-faced breast cancer tissue sections.

\section{Conclusions}

In conclusion, lymphovascular invasion and to a lesser extent microvessel density add prognostic value to the established clinical pathological features in patients with primary operable invasive breast cancer.

\section{Competing interests}

The authors declare that they have no competing interests.

\section{Authors' contributions}

The authors' responsibilities were as follows- ZMAM did the work and wrote the manuscript: DCMCM and JJG provided significant intellectual input and advice in the statistical analysis: DCMCM, JJG and JE provided significant intellectual input and advice in the writing of manuscript: JD prepared the basic database: EM and CO marked the slides and made TMA. All authors read and approved the final manuscript.

\section{Acknowledgements}

The authors gratefully acknowledge funding from Libyan government and the Think Pink charity for raising the money to fund the purchase of image analysis system.

\section{Author details}

${ }^{1}$ Academic Unit of Surgery, College of Medical, Veterinary and Life of Sciences, Royal Infirmary, University of Glasgow, Glasgow G31 2ER, UK ${ }^{2}$ University Departments of Pathology, Faculty of Veterinary Medicine, Omar Almukhtar University, Al bayda, PO Box 919, Libya. ${ }^{3}$ Unit of Experimental 
Therapeutics, Institute of Cancer, College of Medical, Veterinary and Life Sciences, Royal and Western Infirmary, University of Glasgow, Glasgow G11 6NT, UK. ${ }^{4}$ University Department of Pathology, College of Medical, Veterinary and Life of Sciences, Royal and Western Infirmaries, University of Glasgow, Glasgow G11 6NT, UK. ${ }^{5}$ Department of Surgery, Western Infirmary, Glasgow G11 6NT, UK.

Received: 18 July 2012 Accepted: 21 November 2013

Published: 25 November 2013

\section{References}

1. Parkin DM, Bray F, Ferlay J, Pisani P: Estimating the world cancer burden: Globocan 2000. Int J Cancer 2001, 94:153-156.

2. Cancerstats. http:info.cancerresearchuk.org/cancerstats/types/breast/ incidence. Accessed June 2012

3. McGuire WL: Breast cancer prognostic factors: evaluation guidelines. $J$ Natl Cancer Inst 1991, 83:154-155.

4. Lal P, Tan LK, Chen B: Correlation of HER-2 status with estrogen and progesterone receptors and histologic features in 3,655 invasive breast carcinomas. Am J Clin Pathol 2005, 123:541-546.

5. Schnitt SJ: Traditional and newer pathologic factors. J Natl Cancer Inst Monogr 2001, 30:22-26.

6. Anderson WF, Chu KC, Chatterjee N, Brawley O, Brinton LA: Tumor variants by hormone receptor expression in white patients with node-negative breast cancer from the surveillance, epidemiology, and end results database. J Clin Onco 2001, 19:18-27.

7. Bardou VJ, Arpino G, Elledge RM, Osborne CK, Clark GM: Progesterone receptor status significantly improves outcome prediction over estrogen receptor status alone for adjuvant endocrine therapy in two large breast cancer databases. J Clin Onco 2003, 21:1973-1979.

8. Crowe JPJ, Gordon NH, Hubay CA, et al: Estrogen receptor determination and long term survival of patients with carcinoma of the breast. Surg Gynecol Obstet 1991, 173:273-278.

9. Dunnwald LK, Rossing MA, Li Cl: Hormone receptor status, tumor characteristics, and prognosis: a prospective cohort of breast cancer patients. Breast Cancer Res 2007, 9:R6.

10. Shapiro $C L$, Recht $A$ : Side effects of adjuvant treatment of breast cancer. N Engl J Med 2001, 344:1997-2008.

11. Goldhirsch A, Wood WC, Gelber RD, et al: Progress and promise: highlights of the international expert consensus on the primary therapy of early breast cancer 2007. Ann Oncol 2007, 18:1133-1144.

12. Jung SY, Han W, Lee JW, et al: Ki-67 expression gives additional prognostic information on St. Gallen 2007 and Adjuvant! Online risk categories in early breast cancer. Ann Surg Oncol 2009, 16:1112-1121.

13. Viale G, Giobbie-Hurder A, Regan MM, et al: Prognostic and predictive value of centrally reviewed Ki-67 labeling index in postmenopausal women with endocrine-responsive breast cancer: results from Breast International Group Trial 1-98 comparing adjuvant tamoxifen with letrozole. J Clin Onco 2008, 26:5569-5575.

14. de Azambuja E, Cardoso F, de Castro GJ, et al: Ki-67 as prognostic marker in early breast cancer: a meta-analysis of published studies involving 12,155 patients. Br J Cancer 2007, 96:1504-1513.

15. Yerushalmi R, Woods R, Ravdin PM, Hayes MM, Gelmon KA: Ki67 in breast cancer: prognostic and predictive potential. Lancet Oncol 2010, 11:174-183

16. Al Murri AM, Hilmy M, Bell J, et al: The relationship between the systemic inflammatory response, tumour proliferative activity, T-lymphocytic and macrophage infiltration, microvessel density and survival in patients with primary operable breast cancer. $\mathrm{Br} J$ Cancer 2008, 99:1013-1019

17. Rampaul RS, Pinder SE, Elston CW, Ellis IO: Prognostic and predictive factors in primary breast cancer and their role in patient management: The Nottingham Breast Team. Eur J Surg Oncol 2001, 27:229-238.

18. Cianfrocca M, Goldstein LJ: Prognostic and predictive factors in earlystage breast cancer. Oncologist 2004, 9:606-616

19. Mohammed RA, Martin SG, Gill MS, Green AR, Paish EC, Ellis IO: Improved methods of detection of lymphovascular invasion demonstrate that it is the predominant method of vascular invasion in breast cancer and has important clinical consequences. Am J Surg Pathol 2007, 31:1825-1833.

20. Mohammed RA, Martin SG, Mahmmod AM, et al: Objective assessment of lymphatic and blood vascular invasion in lymph node-negative breast carcinoma: findings from a large case series with long-term follow-up. J Pathol 2011, 223:358-365.

21. Pinder SE, Ellis IO, Galea M, O'Rouke S, Blamey RW, Elston CW: Pathological prognostic factors in breast cancer. III. Vascular invasion: relationship with recurrence and survival in a large study with long-term follow-up. Histopathology 1994, 24:41-47.

22. Rakha EA, Martin $\mathrm{S}$, Lee AH, et al: The prognostic significance of lymphovascular invasion in invasive breast carcinoma. Cancer 2012, 118:3670-3680.

23. Gasparini G, Weidner N, Bevilacqua P, et al: Tumor microvessel density, p53 expression, tumor size, and peritumoral lymphatic vessel invasion are relevant prognostic markers in node-negative breast carcinoma. J Clin Onco 1994, 12:454-466.

24. Kato T, Kameoka S, Kimura T, Nishikawa T, Kobayashi M: The combination of angiogenesis and blood vessel invasion as a prognostic indicator in primary breast cancer. Br J Cancer 2003, 88:1900-1908.

25. Lee AK, DeLellis RA, Silverman ML, Heatley GJ, Wolfe HJ: Prognostic significance of peritumoral lymphatic and blood vessel invasion in node-negative carcinoma of the breast. J Clin Onco 1990, 8:1457-1465.

26. Goldhirsch A, Glick JH, Gelber RD, Coates AS, Thürlimann B, Senn HJ: Meeting highlights: international expert consensus on the primary therapy of early breast cancer 2005. Ann Oncol 2005, 16:1569-1583.

27. Lee AH, Pinder SE, Macmillan RD, et al: Prognostic value of lymphovascular invasion in women with lymph node negative invasive breast carcinoma. Eur J Cancer 2006, 42:357-362.

28. Freedman GM, Li T, Polli LV, et al: Lymphatic space invasion is not an independent predictor of outcomes in early stage breast cancer treated by breast-conserving surgery and radiation. Breast J 2012, 18:415-419.

29. Folkman J: What is the evidence that tumors are angiogenesis dependent? J Natl Cancer Inst 1990, 82:4-6.

30. Weidner N, Semple JP, Welch WR, Folkman J: Tumor angiogenesis and metastasis-correlation in invasive breast carcinoma. N Engl J Med 1991, 324:1-8.

31. Longatto FA, Lopes JM, Schmitt FC: Angiogenesis and breast cancer. J Oncol 2010, 2010:1-7.

32. Bevilacqua $P$, Barbareschi $M$, Verderio $P$, et al: Prognostic value of intratumoral microvessel density, a measure of tumor angiogenesis, in node-negative breast carcinoma-results of a multiparametric study. Breast Cancer Res Treat 1995, 36:205-217.

33. Bosari S, Lee AK, DeLellis RA, Wiley BD, Heatley GJ, Silverman ML: Microvessel quantitation and prognosis in invasive breast carcinoma. Hum Pathol 1992, 23:755-761

34. Fox SB, Leek RD, Weekes MP, Whitehouse RM, Gatter KC, Harris AL: Quantitation and prognostic value of breast cancer angiogenesis: comparison of microvessel density, Chalkley count, and computer image analysis. J Pathol 1995, 177:275-283.

35. Ogawa Y, Chung YS, Nakata B, et al: Microvessel quantitation in invasive breast cancer by staining for factor VIII-related antigen. Br J Cancer 1995, 71:1297-1301

36. Toi M, Kashitani J, Tominaga T: Tumor angiogenesis is an independent prognostic indicator in primary breast carcinoma. Int J Cancer 1993, 55:371-374

37. Weidner N, Folkman J, Pozza F, et al: Tumor angiogenesis: a new significant and independent prognostic indicator in early-stage breast carcinoma. J Natl Cancer Inst 1992, 84:1875-1887.

38. Martin L, Green B, Renshaw C, et al: Examining the technique of angiogenesis assessment in invasive breast cancer. $\mathrm{Br} J$ Cancer 1997 76:1046-1054

39. Foekens JA, Peters HA, Grebenchtchikov N, et al: High tumor levels of vascular endothelial growth factor predict poor response to systemic therapy in advanced breast cancer. Cancer Res 2001, 61:5407-5414

40. Manders P, Beex LV, Tjan-Heijnen VC, Span PN, Sweep CG: Vascular endothelial growth factor is associated with the efficacy of endocrine therapy in patients with advanced breast carcinoma. Cancer 2003, 98:2125-2132

41. Goulding H, Abdul Rashid NF, Robertson JF, et al: Assessment of angiogenesis in breast carcinoma: an important factor in prognosis? Hum Pathol 1995, 26:1196-1200.

42. Van Hoef ME, Knox WF, Dhesi SS, Howell A, Schor AM: Assessment of tumour vascularity as a prognostic factor in lymph node negative invasive breast cancer. Eur J Cancer 1993, 29A:1141-1145. 
43. Protopapa E, Delides GS, Revesz L: Vascular density and the response of breast carcinomas to mastectomy and adjuvant chemotherapy. Eur J Cancer 1993, 29A:1391-1393.

44. Tovey SM, Dunne B, Witton CJ, Cooke TG, Bartlett JMS: HER4 in breast cancer: comparison of antibodies against intra- and extra-cellular domains of HER4. Breast Cancer Res 2006, 8:R19.

45. Mohammed ZM, Edwards J, Orange C, et al: Breast cancer outcomes by steroid hormone receptor status assessed visually and by computer image analysis. Histopathology 2012, 61:283-292.

46. Mohammed ZM, Going JJ, McMillan DC, et al: Comparison of visual and automated assessment of HER2 status and their impact on outcome in primary operable invasive ductal breast cancer. Histopathology 2012. 61:675-684.

47. Mohammed ZM, MCMillan DC, Elsberger B, et al: Comparison of Visual and automated assessment of Ki-67 proliferative activity and their impact on outcome in primary operable invasive ductal breast cancer. $\mathrm{Br} J$ Cancer 2012, 106:383-388

48. Ovcaricek T, Frkovic SG, Matos E, Mozina B, Borstnar S: Triple negative breast cancer - prognostic factors and survival. Radiol Oncol 2011, 45:46-52

49. Camp RL, Rimm EB, Rimm DL: A high number of tumor free axillary lymph nodes from patients with lymph node negative breast carcinoma is associated with poor outcome. Cancer 2000, 88:108-113.

50. Kim SH, Simkovich-Heerdt A, Tran KN, Maclean B, Borgen PI: Women 35 years of age or younger have higher locoregional relapse rates after undergoing breast conservation therapy. J Am Coll Surg 1998, 187:1-8.

51. Hayes DF, Isaacs C, Stearns V: Prognostic factors in breast cancer: current and new predictors of metastasis. J Mammary Gland Biol Neoplasia 2001, 6:375-392.

52. Allen M, Louise JJ: Jekyll and Hyde: the role of the microenvironment on the progression of cancer. J Pathol 2011, 223:162-176.

doi:10.1186/1472-6890-13-31

Cite this article as: Mohammed et al:: The relationship between lymphovascular invasion and angiogenesis, hormone receptors, cell proliferation and survival in patients with primary operable invasive ductal breast cancer. BMC Clinical Pathology 2013 13:31.

\section{Submit your next manuscript to BioMed Central and take full advantage of:}

- Convenient online submission

- Thorough peer review

- No space constraints or color figure charges

- Immediate publication on acceptance

- Inclusion in PubMed, CAS, Scopus and Google Scholar

- Research which is freely available for redistribution 